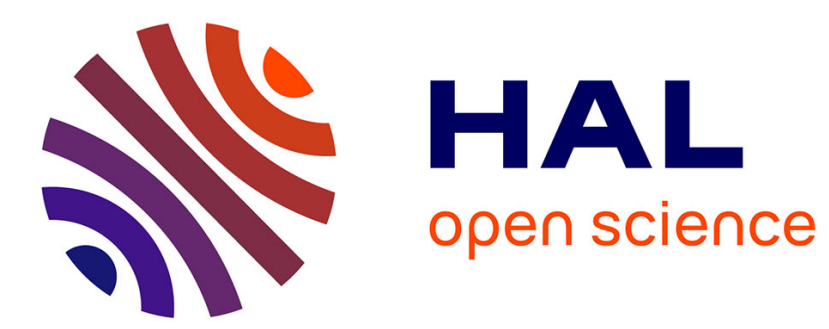

\title{
Le serpenteau transhumaniste
}

Olivier Rey

\section{- To cite this version:}

Olivier Rey. Le serpenteau transhumaniste. Pardès, 2020, Le transhumanisme à l'epreuve de la Bible

- Israël et le message du judaïsme, 63, pp.57-66. halshs-02908295

\section{HAL Id: halshs-02908295 \\ https://shs.hal.science/halshs-02908295}

Submitted on 28 Jul 2020

HAL is a multi-disciplinary open access archive for the deposit and dissemination of scientific research documents, whether they are published or not. The documents may come from teaching and research institutions in France or abroad, or from public or private research centers.
L'archive ouverte pluridisciplinaire HAL, est destinée au dépôt et à la diffusion de documents scientifiques de niveau recherche, publiés ou non, émanant des établissements d'enseignement et de recherche français ou étrangers, des laboratoires publics ou privés. 


\title{
« Le transhumanisme à l'épreuve de la Bible »
}

\author{
Journée d'étude organisée par l'Université populaire du judaïsme \\ Paris, Forum 104, 19 mai 2019
}

Un nouveau discours enfle dans le débat public qui annonce le "transhumain », le "posthumain», l'«homme augmenté», le «cyborg», en tout cas «la fin de l'humain», pour reprendre le titre d'un livre.

Quelle est la nature de ce nouvel évangile, de ce nouveau « Grand récit » sur fond de tous les mondialismes et de tous les progressismes ? Quel est le sens de son apparition aujourd'hui ? Puisqu'il promeut une refondation de l'humain, il nous intime la nécessité de revenir aux fondements, c'est-à-dire au Livre de la Genèse et à sa construction de l'humain, ne serait-ce que parce que les tenants de la déshumanisation objective de l'homme s'insurgent souvent contre son supposé enseignement, son naturalisme, son moralisme, son déterminisme...

Que recèle la philosophie du Livre de la Genèse face à ces enjeux et l'appel de l'infini qu'ils donnent à entendre? Que pourrait receler la Genèse d'inattendu, d'inédit pour le débat contemporain? 


\title{
Le serpenteau transhumaniste ${ }^{1}$
}

\author{
Olivier REY
}

À propos du transhumanisme, une chose mérite d'être d'emblée rappelée et soulignée : ses promesses les plus spectaculaires - l'immortalité, la symbiose entre l'homme et la machine, le téléchargement du psychisme sur ordinateur et autres merveilles - ne sont pas sérieuses. Elles ne sont que conceptions d'ingénieurs informatiques plaqués sur du vivant. Lorsque les premiers calculateurs électroniques ont été mis au point, on a parlé de cerveaux électroniques. Ces calculateurs sont devenus ordinateurs, et parallèlement, la métaphore s'est retournée : c'est le cerveau qui a été comparé, et même assimilé à un ordinateur - «un ordinateur fait de viande », pour reprendre une expression prisée par le propagandiste Laurent Alexandre. D'où l'idée que le cerveau peut être relié directement à la machine, et upgradé comme elle.

À l'appui de leur programme fantaisiste, les transhumanistes opposent l'avis de quelques scientifiques éminents qui appuient leurs dires. L'éminence scientifique n'est cependant pas une garantie de sérieux, surtout quand les sommités de la science s'aventurent en dehors de leur domaine de compétence. Étant donné le nombre de scientifiques de par le monde, il n'est pas étonnant qu'il s'en trouve parmi eux pour tenir des propos insensés, avec d'autant plus d'aplomb que leur supériorité dans un certain secteur leur a fait perdre le sens de l'humilité, et que leurs vaticinations peuvent leur procurer un avantage de notoriété, voire financier si leur discours sert de caution à des intérêts économiques. Les intérêts économiques justement : l'appui que de grandes firmes donnent au projet transhumaniste n'est-il pas la meilleure preuve que celui-ci est crédible? De l'argent irait-il soutenir ce qui n'est pas réalisable ? Oui, cela est parfaitement possible. Non seulement parce que les investisseurs sont sujets à commettre de lourdes erreurs, mais encore parce que le soutien à un projet peut être déterminé non par la crédibilité qui lui est accordée, mais par l'utilité que le projet en question, quand bien même il n'aboutirait jamais, revêt pour le développement actuel des affaires.

\footnotetext{
${ }^{1}$ Un certain nombre des arguments qui figurent dans ce texte sont empruntés aux ouvrages Leurre et malheur $d u$ transhumanisme (Desclée de Brouwer, 2018) et Une question de taille (Stock, 2014).

Le présent texte a été publié dans la revue Pardès. Études et cultures juives, $\mathrm{n}^{\circ} 63:$ « Le transhumanisme à l'épreuve de la Bible - Israël et le message du judaïsme », Paris, Éditions In Press, 4 mars 2020, p. 57-66 (ISSN 0295-5652 ; ISSN en ligne 2271-1880 ; ISBN 978-2-84835-561-0).
} 
Pour comprendre le rôle que le transhumanisme est ici amené à jouer, il faut considérer la situation historique particulière dans laquelle nous nous trouvons. Certains ont parlé, dans les années 1970, d'un changement d'époque - le passage de la modernité à la postmodernité. Depuis, la notion de postmodernité a connu une rapide fortune. Pour comprendre de quoi il retourne il est bon de se reporter à l'ouvrage de Jean-François Lyotard, paru en 1979, et intitulé précisément La Condition postmoderne ${ }^{2}$. Selon Lyotard, l'élément le plus déterminant dans le passage de la modernité à la postmodernité est un changement dans le rapport à la science. Schématiquement, deux grands récits ont porté le développement de la science au cours des derniers siècles. Le premier, marié à des aspirations politiques, est le récit de l'émancipation: en dissipant les superstitions et en dévoilant le monde dans sa vérité, la science moderne devait permettre aux hommes de se libérer de leurs anciennes tutelles, de prendre leur destin en main, d'accéder à l'autonomie et de soulager leurs peines en transformant le monde à leur gré. Le second grand récit, qui a présidé à l'essor et à la réussite extraordinaire de l'université allemande au XIX ${ }^{\mathrm{e}}$ siècle, est le récit spéculatif : la science était conçue comme accomplissement de la vie de l'esprit, réalisation de l'Esprit universel. Le $\mathrm{XX}^{\mathrm{e}}$ siècle s'est révélé cruel avec ces deux grands récits. Il a montré que la science peut aussi bien servir à l'asservissement de l'homme qu'à son émancipation, et n'offre aucune garantie contre la nuit de l'esprit. Le lien automatique entre le progrès scientifique et le progrès humain s'est défait. Prenant acte de l'épuisement des deux grands récits qui accompagnaient et soutenaient la science moderne, Lyotard a décrit le passage à un nouveau régime, postmoderne, du savoir scientifique, au sein duquel les critères d'efficacité sont devenus déterminants, au point de prendre le pas sur toutes les autres considérations, y compris l'établissement de la vérité. Autre façon de le formuler: nous sommes passés d'une perspective de progrès, à une apologie de la simple innovation - sans que l'on sache si celleci constitue, ou non, un progrès.

Cela étant, des fractions de plus en plus importantes de la population se mettent à douter. Vaut-il bien la peine de s'épuiser à suivre le rythme endiablé de l'innovation, si celleci ne constitue pas un véritable progrès ? Ce genre de perplexité, évidemment, est mauvais pour la consommation, pour la croissance, pour les affaires. On comprend alors le rôle que les promesses les plus fantastiques du transhumanisme sont appelées à jouer : il s'agit d'arracher l'adhésion de populations de plus en plus dubitatives à la poursuite de la dynamique d'artificialisation et de technologisation de l'existence. La perte de confiance dans le progrès doit être compensée par une inflation de ce que l'innovation est censée apporter : à savoir la réalisation des fantasmes de toute-puissance de l'individu.

De ce point de vue, le transhumanisme joue sur les mêmes ressorts que le serpent de la Genèse. N'étant pas hébraïsant, je ne pourrai malheureusement que me référer aux traductions grecques, latines et françaises du texte biblique. Quand Dieu se propose de créer l'homme, les

\footnotetext{
${ }^{2}$ La Condition postmoderne. Rapport sur le savoir, Paris, Minuit, coll. « Critique », 1979.
} 
deux mots qui caractérisent le rapport d'affinité entre le Créateur et sa créature sont traduits, en grec, par eikôn et homoiôsis, en latin par imago et similitudo, en français par «image » et « ressemblance ». «Dieu dit : "Faisons l'homme à notre image, comme notre ressemblance" » (Gn 1, 26). Que signifie ici l'image ? La ressemblance ? La différence entre les deux, s'il y en a une ? Et le fait que lorsque Dieu crée effectivement l'homme - «Dieu créa l'homme à son image, à l'image de Dieu il le créa, homme et femme il les créa » $(\mathrm{Gn} \mathrm{1,27)}$-, il n'est plus question que d'image, la ressemblance n'étant pas mentionnée ? D'innombrables commentateurs ont donné à ces questions d'innombrables réponses, dont il serait vain de seulement songer à dresser un panorama. Je me contenterai donc ici de suivre un certain fil exégétique des traditions grecque et latine.

Premièrement, le fait que l'homme soit «à l'image » de Dieu est un élément constitutif, inamissible. Deuxièmement, ce donné indique aussi une vocation : le kat' eikona en grec, ad imaginem en latin, «à l'image » en français est un appel à la ressemblance, qui peut être effective ou non - tout le monde sait qu'une image peut être plus ou moins ressemblante. Dans les chapitres 2 et 3 de la Genèse, deux voies sont proposées pour accomplir cette ressemblance.

Au chapitre 2, un interdit est formulé - l'interdit de manger du fruit de l'arbre de la connaissance du bien et du mal. Cet interdit a beau se présenter, par rapport à la parole du premier chapitre - «Je vous donne toutes les herbes portant semence, qui sont sur toute la surface de la terre, et tous les arbres qui ont des fruits portant semence: ce sera votre nourriture » $(\mathrm{Gn} 1,29)$-, comme une restriction au don initial, il s'agit en réalité d'un don supplémentaire. En effet, que les fruits d'un arbre unique soient soustraits à la consommation n'engendre aucune privation (l'abondance des autres fruits est plus que suffisante à la nourriture); et l'interdit, au lieu d'amoindrir les facultés humaines, les accroît, en apprenant aux hommes que leur puissance ne consiste pas seulement à faire, mais aussi à ne pas faire. Dieu est dit tout-puissant parce que ce qu'il veut, il le peut. Mais il ne veut pas n'importe quoi : en lui, volonté, sagesse, amour et puissance vont toujours de pair - la puissance de Dieu est « ordonnée ». La situation de l'homme est différente. D'un côté, il arrive à l'être humain de vouloir sans pouvoir, de l'autre, son vouloir et son agir ne sont pas nécessairement ordonnés à la sagesse et à l'amour. Aussi infime soit la puissance humaine en comparaison de la puissance divine, il se produit avec elle ce qui n'advient jamais avec la puissance divine, à savoir que la volonté et la puissance d'agir excèdent ce que la sagesse et l'amour inspirent et recommandent. De ce fait, l'être humain doit s'abstenir de certains actes qui lui sont néanmoins possibles : c'est précisément ce que réclame de lui l'interdit. Par-delà l'abîme qui sépare la puissance finie de l'homme de la puissance infinie de Dieu, la première peut ressembler à la seconde en ce qu'elle sera, comme elle, ordonnée.

La seconde voie de la ressemblance à Dieu est proposée au chapitre 3 de la Genèse, par le serpent. Le discours du serpent présente Dieu comme un potentat, jaloux de sa supériorité, et l'interdit non comme un don supplémentaire à sa créature, mais comme un moyen d'assurer 
et préserver sa domination. Selon le serpent, s'abstenir de certains actes n'est pas pour l'homme le moyen, en ordonnant sa puissance, de ressembler à son Créateur, mais un renoncement à lui ressembler. Quand l'être humain se laisse pénétrer par ce genre d'idées, il n'a plus de cesse que de manger les fruits de l'arbre défendu. Ces fruits n'avaient aucune vertu particulière : contrairement aux dires du serpent, en manger n'a pas rendu l'homme et le femme omniscients. Loin de là : enfreindre l'interdit n'a pas augmenté, mais altéré la connaissance. En quel sens ? Il est significatif que l'avertissement divin, portant sur des fruits qu'il ne faut pas manger, ait été formulé juste avant la création d'Ève. L'être humain doit admettre une limite à sa volonté d'emprise pour appréhender et respecter en l'autre un vis-àvis, être à même de le connaître de la façon dont il mérite d'être connu ${ }^{3}$. Ce qui importait donc, c'était la limite que les humains acceptaient de mettre à leur volonté d'emprise. L'acte transgresseur a procédé d'une conception fausse de Dieu, implantant dans leur imaginaire un désir d'omniscience, un appétit de maîtrise totale. Dieu constate : «Voilà que l'homme est devenu comme l'un de nous, pour connaître le bien et le mal ! » (Gn 3,22). C'est-à-dire : dans le but de tout connaître, l'homme est entré en rivalité mimétique avec nous. L'inverse est également vrai : c'est d'une rivalité mimétique avec Dieu que procède une volonté d'emprise totale. En voulant être pareil à Dieu, l'homme n'a pas effacé la différence d'essence qui l'en sépare, mais il a perdu la ressemblance dont, malgré cette différence, il est capable. En cédant à une fausse idée de la ressemblance, il a déformé en lui l'image de Dieu. Rupert de Deutz, théologien liégeois qui vécut à la charnière des $\mathrm{XI}^{\mathrm{e}}$ et XII ${ }^{\mathrm{e}}$ siècles, imagine Satan qui se réjouit : «Dieu n'a pas fait l'homme à sa ressemblance : c'est moi qui l'ai fait à la mienne ${ }^{4}$ ! »

Il en va de même, toutes proportions gardées, avec les promesses du transhumanisme. Augmentez-vous, et vous sortirez de la condition humaine, vous serez comme des dieux. Adam et Ève, après avoir mangé du fruit, ne deviennent pas des dieux, ils deviennent esclaves du péché. Les «augmentés » ne seront pas davantage des dieux, ils seront ravalés au rang d'animaux monitorés.

Il n'est, pour illustrer ce point, que de considérer la politique de Google, la firme qui soutient avec le plus d'ardeur le discours transhumaniste. Au cours des dernières années, Google a passé alliance avec les plus grandes entreprises pharmaceutiques (Pfizer, Novartis, Sanofi...). Pour quelles raisons ? Il s'agit de marier l'extraordinaire capacité de Google à collecter sur ses serveurs et à traiter par ses logiciels de gigantesques masses de données, et la faculté de l'industrie pharmaceutique à fournir à tout un chacun, sur la base des corrélations établies au sein de ces données, des produits prétendument adaptés à son cas. Google, du reste, entend à terme tirer parti directement des amas d'informations qu'elle recueille. Verily, sa filiale de santé, a annoncé en 2017 qu'elle allait suivre une cohorte de 10000 personnes dont elle séquencera le génome et dont, pendant au moins quatre ans, elle surveillera

\footnotetext{
${ }^{3}$ À ce sujet voir Marie Balmary, La Divine Origine. Dieu n'a pas créé l'homme, Grasset, 1993, chap. III.

${ }^{4}$ De victoria Verbi Dei, livre II, chap. 8 (Patrologia latina 169, 1250BC).
} 
(notamment par l'intermédiaire d'une «montre d'étude » (Study Watch) pourvue de capteurs, que chaque participant portera en permanence au poignet), quantité de paramètres biologiques et cliniques. Une multitude de tests et de mesures seront effectués en parallèle, auxquels s'ajouteront renseignements sur le cadre et le mode de vie. L'objectif déclaré est d'établir une « carte de la santé humaine », de «mieux comprendre la transition entre la bonne santé et la maladie », d' « identifier des facteurs de risque additionnels pour les maladies » ainsi que des marqueurs biologiques avant-coureurs de pathologies, qui pourraient ainsi être prévenues avant leur déclenchement ${ }^{5}$. Se prépare de la sorte une pharmacisation tous azimuts de l'existence ${ }^{6}$ qui, au nom de la santé, induira une préoccupation permanente, parfaitement contradictoire avec la véritable santé qui est un état où, précisément, celle-ci n'est pas une préoccupation.

L'état de complet bien-être physique, mental et social est rien moins qu'assuré, mais l'état de complète dépendance à l'égard de ceux qui recommandent, fabriquent et délivrent les traitements, quant à lui, ne fait aucun doute. C'est à cela que sert, pour une bonne part, la rhétorique de la «médecine personnalisée » et de l'«augmentation». L'homme comme vivant politique doit laisser place à l'homme comme animal monitoré, branché en permanence sur le système qui le surveille et le guide. Beaucoup de ménages souffrent aujourd'hui de la part sans cesse croissante dans leur budget des dépenses dites contraintes : il devient toujours plus onéreux d'être simplement en mesure d'exister dans l'espace social. Imaginons ce qu'il en serait si en plus nous devions financer nos «augmentations », leur entretien et leurs mises à jour permanentes. Et plus question, alors, de se rebeller, façon Gilets jaunes : il suffirait aux maîtres du système, pour juguler toute contestation, de désactiver à distance les fonctions implantées, sans lesquelles on aurait désappris à vivre. En fait d'émancipation, c'est le retour à la dépendance du nourrisson, moyennant le passage de la mère au réseau, des tétons aux connexions.

Pour terminer, je voudrais évoquer brièvement en quoi l'esprit du transhumanisme s'apparente au gnosticisme des premiers siècles du christianisme, et en quoi il en diffère. On a qualifié de gnostiques un certain nombre d'hérésies chrétiennes qui ont fleuri aux $\mathrm{II}^{\mathrm{e}}$ et III ${ }^{\mathrm{e}}$ siècles et qui, dans leur diversité, avaient pour point commun un dualisme radical, qui les amenait à distinguer et opposer d'un côté la puissance démiurgique de l'Ancien Testament, de l'autre le Dieu rédempteur qui s'est incarné en Jésus-Christ, qui les amenait à condamner le monde corporel et à concevoir le salut comme un affranchissement complet de l'esprit vis-àvis de sa prison charnelle, grâce à une certaine connaissance (la gnose). Saint Irénée, à la suite

\footnotetext{
${ }^{5}$ Voir https://www.projectbaseline.com/ et https://verily.com/projects/precision-medicine/baseline-study/.

${ }^{6}$ Sur cette question, voir l'article de Nicolas Le Dévédec et Johanne Collin, « Le médicament augmenté. L'usage du médicament dans les discours transhumanistes et ses significations sociales », Journal international de bioéthique et d'éthique des sciences, vol. 29, n³-4, 2018, p. 93-108.
} 
de saint Paul, s'est attaché à distinguer la «vraie gnose », alliance de la foi et de la raison pour connaître les choses divines, de ce qu'il qualifiait de « gnose au nom menteur » (auquel fait référence l'adjectif «gnostique »), doctrines opposant l'esprit à la création et plaçant le salut dans une «excarnation ». Pour le christianisme orthodoxe il n'y a pas, de l'Ancien au Nouveau Testament, opposition mais accomplissement; et parce que, selon la Genèse, l'homme n'est pas un esprit prisonnier de la glaise, mais de la glaise à laquelle a été insufflée une haleine de vie, le salut passe non par une annulation de la chair, mais par sa résurrection glorieuse - il n'y aurait pas de sens à considérer l'esprit présent en chaque être humain indépendamment d'un corps dont il est le principe d'animation. Cependant, même vaincues, les tendances gnostiques (ou « gnosticistes ») n'ont pas disparu.

À dire vrai elles ne sauraient s'éteindre, car leurs racines plongent dans des données fondamentales de la condition humaine. Le petit enfant, dans son Hilflosigkeit, sa dépendance absolue et prolongée à l'égard des adultes qui prennent soin de lui (les Nebenmenschen), attribue à ces adultes une puissance qui dépasse de loin ce qu'elle est en réalité. Dès lors que, dans un grand nombre de cas, ses parents comblent ses attentes, soulagent ses peines et lui procurent du plaisir, il sera enclin à leur attribuer une capacité à toujours le satisfaire et, de ce fait, à attribuer à leur mauvaise volonté tous les malaises qu'il lui arrive d'éprouver et dont ils ne le délivrent pas. Cette ambivalence des figures parentales est profondément troublante: comment les mêmes êtres peuvent-ils être à la fois la source de tout bien et l'origine, réelle ou imaginaire, de multiples frustrations ? Il y a un tel contraste, pour l'enfant, entre le parent bénédiction et le parent frustrateur, que pour s'y reconnaître il est enclin à cliver les représentations parentales. «La perception de la double nature des parents amène l'enfant à découvrir qu'ils ne sont pas simplement la projection de ses propres désirs. La défense classique contre cette découverte - un des mécanismes classiques du déni - est de diviser les images parentales en bonnes et mauvaises images. Le nourrisson, dans ses fantasmes, dissocie chez les adultes qui prennent soin de lui les aspects frustrateurs des aspects qui procurent du plaisir. C'est ainsi qu'il invente des images idéalisées de seins et qu'il les fait cohabiter avec des images d'une autorité omnipotente, menaçante et destructrice, maternelle ou paternelle un vagin dévorant, un pénis ou un sein castrateur ${ }^{7}$.» Il y a la bonne mère et la mauvaise mère, le bon père et le mauvais père. Cette dernière opposition est mise en scène, de façon très pure, dans la nouvelle de Philip K. Dick «Le père truqué » [The Father-Thing], où des envahisseurs extra-terrestres remplacent le «bon père» par le «mauvais père ». Dans la notice de présentation de la nouvelle, Dick écrit: «Quand j'étais tout petit, j'avais constamment l'impression que mon père était deux personnes, une bonne et une mauvaise. Il $\mathrm{y}$ avait toujours un moment où le bon père disparaissait et où le mauvais prenait sa place. Sans

\footnotetext{
${ }^{7}$ Christopher Lasch, La Culture du narcissisme. La vie américaine à un âge de déclin des espérances [1979], trad. Michel L. Landa, Paris, Flammarion, coll. « Champs », 2006, p. 303-304.
} 
doute est-ce une sensation assez répandue chez les enfants ${ }^{8}$. » Christopher Lasch situe dans un tel clivage l'origine de tous les manichéismes. «Le dualisme religieux institutionnalise ces défenses primitives et régressives en séparant rigoureusement les images nourrissantes et miséricordieuses des images de création, de jugement et de châtiment. La version particulière du dualisme connue sous le nom de gnosticisme, qui a fleuri dans le monde hellénistique aux $\mathrm{II}^{\mathrm{e}}, \mathrm{III}^{\mathrm{e}}$ et $\mathrm{IV}^{\mathrm{e}}$ siècles après Jésus-Christ, a conduit ce déni à ses conclusions les plus radicales. Elle a condamné le monde matériel dans son ensemble comme création de puissances ténébreuses et diaboliques. Le gnosticisme a donné une forme mythologique - souvent touchante et expressive - aux fantasmes qui permettent d'entretenir l'illusion archaïque de ne faire qu'un avec un monde répondant totalement à nos souhaits et désirs. En niant qu'un créateur bienveillant ait pu façonner un monde qui contient à la fois la souffrance et la gratification, le gnosticisme a perpétué l'espoir d'un retour à une condition spirituelle dans laquelle ces expériences [mélangées] seraient inconnues ${ }^{9}$.» En réalité, ce sont les mêmes parents qui tantôt comblent, tantôt font défaut ou s'opposent, c'est le même monde qui est à la fois source de plaisir et de souffrance. Contre le gnosticisme, le christianisme orthodoxe affirme l'unité du Dieu créateur de la Genèse et du Dieu rédempteur incarné dans la personne du Christ.

Mais l'inspiration gnostique, tenue en respect, a trouvé dans le contexte moderne un terrain particulièrement propice à sa résurgence. Le progrès technique en effet, qui pendant un certain temps a conféré aux individus une autonomie croissante, passé un certain seuil s'est mis au contraire à défaire cette autonomie, dans la mesure où plus rien n'est possible à l'individu en dehors de son branchement permanent sur un gigantesque système technologique: la dépendance que les capacités techniques faisaient diminuer, le déchaînement technologique la reconduit sous une nouvelle forme. Et plus cette dépendance devient écrasante, plus elle favorise, à titre de compensation, les fantasmes mégalomaniaques de surpuissance - qui vont aller s'investir dans cela même qui fait croître aujourd'hui la dépendance, à savoir la frénésie technologique. Le gnosticisme ancien était religieux - il entendait, par la connaissance du vrai Dieu, libérer l'esprit de toute attache au monde matériel, abandonné aux forces mauvaises. Le gnosticisme moderne, quant à lui, entend, par la technologie, soumettre le monde à l'esprit. La dynamique de transformation du monde par la technologie moderne ne peut être vraiment saisie si l'on ignore cette dimension spirituelle : le gnosticisme moderne, c'est la séparation de l'esprit et de la matière qui devient, au gré des moyens d'action conférés par la technologie, impérialisme de la volonté, impatience devant tout donné, désir illimité de maîtrise et de contrôle.

\footnotetext{
${ }^{8}$ Voir Nouvelles, trad. revues par Hélène Collon, 2 vol., Paris, Denoël, coll. «Lunes d'encre », 2000, t. I, p. 1310-1324 (trad. Alain Dorémieux).

${ }^{9}$ La Culture du narcissisme, op. cit., p. 304.
} 
Selon Eric Voegelin, «l'essence de la modernité consiste en un accroissement du gnosticisme $^{10} »$, à travers une «immanentisation» de ses objectifs. Dans la mesure où le gnosticisme est un spiritualisme, et où la science moderne et la technique qui en est solidaire - cela même que j'appelle technologie, pour la distinguer d'un ancien régime de la technique -, dans la mesure donc mesure où le gnosticisme est un spiritualisme et où science moderne et technologie se donnent généralement pour des matérialismes, on pourrait considérer leur rapprochement comme une contradiction dans les termes. Tel n'est pas le cas, car là même où un certain esprit scientifique moderne récuse les anciens dualismes pour se revendiquer comme moniste, ce prétendu monisme est en réalité un dualisme si radical qu'il en perd la faculté de se reconnaître comme tel. Celui qui, par exemple, affirme que la pensée est un processus purement matériel n'est pas, comme il le prétend, matérialiste, il est en fait tellement idéaliste qu'il ne s'en aperçoit plus. C'est précisément de cette position idéaliste ignorée qu'il peut déclarer que tout est matière. Et quand il prétend que l'esprit naît de la matière, il entend en fait assurer la domination totale de l'esprit en dominant sa propre apparition.

Ce qui est curieux, et effrayant, c'est que ceux aujourd'hui qui imaginent, par la technologie, rompre avec tout ce qui este en l'homme d'archaïque, ne se rendent pas compte qu'ils sont précisément sous l'emprise de motions très archaïques. Lorsque j'étais enfant, une devinette de cour de récréation demandait : qu'est-ce qui est petit, jaune, et qui fait très peur ? La réponse était : un poussin avec une mitraillette. J'ai découvert plus tard que cette blague annonçait quelque chose de notre temps, qui voit le couplage monstrueux de la surpuissance et de l'infantilisme, dans la figure hideuse de l'immature surarmé. Dépasser cette figure, telle serait la véritable augmentation.

\footnotetext{
${ }^{10}$ La Nouvelle Science du politique. Une introduction [1952], trad. Sylvie Courtine-Denamy, Paris, Le Seuil, coll. «L'Ordre philosophique », 2000, p. 183. Hans Jonas ne l'a pas exprimé en des termes aussi tranchés, mais entre ses premiers travaux, sur le phénomène gnostique, et les réflexions qui ont suivi, inspirées par la situation actuelle et ses périls, il existe bel et bien un lien.
} 